\title{
Scapholunate Ligament Reconstruction
}

\author{
Mark Ross, MBBS, FRACS (Orth) ${ }^{1,2,3}$ Jeremy Loveridge, MBBS $^{1,2} \quad$ Kenneth Cutbush, MBBS, FRACS (Orth) ${ }^{1}$ \\ Greg Couzens, MBBS, FRACS (Orth) $)^{1,2}$
}

1 Brisbane Hand and Upper Limb Research Institute, Brisbane, QLD,

Address for correspondence Mark Ross, MBBS, FRACS(ORTH),

Australia

2 Department of Orthopaedic, Princess Alexandra Hospital, Brisbane Hand and Upper Limb Research Institute, 9/259 Wickham

Woolloongabba, QLD, Australia

${ }^{3}$ School of Medicine (Orthopaedic Surgery), The University of

Queensland, Brisbane, QLD, Australia

Terrace, Brisbane, QLD, Australia (e-mail: research@upperlimb.com).

J Wrist Surg 2013;2:110-115.

\begin{abstract}
Background Scapholunate reconstruction poses a challenge to orthopedic surgeons. Materials and Methods Prospective cohort.

Description of Technique Our technique for scapholunate (SL) reconstruction involves ligament reconstruction utilizing a portion of the flexor carpi radialis tendon rerouted via transosseous tunnels across the scaphoid, lunate, and triquetrum (scapholunotriquetral tenodesis). The tendon graft is secured with interference screw fixation into the triquetrum. The philosophy of this new technique is to reduce subluxation and maintain the relationship between scaphoid and lunate by placing a graft through the center of the SL articulation. This graft is then tensioned by passing it centrally through the lunate and triquetrum and secured using an interference screw in the triquetrum. Secondary stabilizers, including the dorsal intercarpal ligament, are then augmented by passing the graft back to the scaphoid, crossing from the triquetrum over the proximal capitate. This further reinforces the translational relationship between the scaphoid and the triquetrum and, therefore, augments stability of the SL articulation.

\section{Keywords}

- scapholunate

- luno-triquetral

- reconstruction

- wrist

- interference screw

- surgical technique

Results We have utilized this technique successfully in over 40 patients since 2009 . We report on a prospective consecutive series of 11 patients with over 12 months follow-up (range 12 to 24 months) demonstrating good early radiological and clinical outcomes. Conclusions In developing this technique, we aimed to take the best features of previously described techniques and address the perceived shortcomings of each. We believe there are several benefits of our technique. Moreover, few other techniques address as many of the aspects of chronic SL instability as our technique does.
\end{abstract}

Reconstruction of chronic scapholunate (SL) ligament pathology remains a significant challenge for hand surgeons. There are several unresolved issues in regard to when reconstruction should be undertaken in preference to repair. This question relates to the difficulties in defining what represents acute, subacute, and chronic SL ligament pathology.

\section{Patients and Methods}

This technique is indicated for a dynamic or reducible (intraoperatively) static SL instability where other forms of SL reconstruction may be considered. It may also offer an augmentation for acute and semi-acute repairs of the SL ligament. It has also been successfully used in acute 
Table 1 Patient demographics and clinical details

\begin{tabular}{|c|c|c|c|c|c|c|c|}
\hline Patient & Age gender & $\begin{array}{l}\text { Insurance } \\
\text { status }\end{array}$ & Occupation & $\begin{array}{l}\text { Mechanism } \\
\text { of injury }\end{array}$ & $\begin{array}{l}\text { SLL } \\
\text { Geisler } \\
\text { Grade }\end{array}$ & $\begin{array}{l}\text { Relevant medical } \\
\text { history prior to SLL } \\
\text { recon }\end{array}$ & Complication \\
\hline 1 & 29 Male & Army & Soldier & Twisting Injury & 4 & None & Nil \\
\hline 2 & 48 Male & WC & $\begin{array}{l}\text { Warehouse } \\
\text { Laborer }\end{array}$ & Fall & 4 & None & Nil \\
\hline 3 & 29 Female & Third Party & Hairdresser & MVA & 4 & $\begin{array}{l}\text { Previous failed closed } \\
\text { treatment of perilu- } \\
\text { nate dislocation }\end{array}$ & Nil \\
\hline 4 & 29 Male & $\begin{array}{l}\text { Professional } \\
\text { Football }\end{array}$ & Footballer & $\begin{array}{l}\text { Fall/twisting } \\
\text { injury }\end{array}$ & 4 & None & Nil \\
\hline 5 & 23 Female & WC & $\begin{array}{l}\text { Customer } \\
\text { Service }\end{array}$ & $\begin{array}{l}\text { Motor vehicle } \\
\text { accident }\end{array}$ & 4 & None & Nil \\
\hline 6 & 35 Male & Private & Baker & Fall & 4 & $\begin{array}{l}\text { Previous scaphoid } \\
\text { fracture } 15 \text { years ago }\end{array}$ & Nil \\
\hline 7 & 40 Male & Private & Manager & Shotgun recoil & 4 & None & Nil \\
\hline 8 & 43 Male & WC & $\begin{array}{l}\text { Manual } \\
\text { Laborer }\end{array}$ & Twisting Injury & 4 & None & Nil \\
\hline 9 & 36 Male & Army & Gunner & $\begin{array}{l}\text { Hyperextension } \\
\text { injury }\end{array}$ & 3 & Previous SLL repair & Nil \\
\hline 10 & 30 Male & Third Party & Builder & Insidious onset & 4 & None & $\begin{array}{l}\text { Rupture following } \\
\text { hyperextension } \\
\text { injury to wrist. } \\
\text { Subsequent partial } \\
\text { wrist fusion } 3 \text { months } \\
\text { post SL recon. }\end{array}$ \\
\hline 11 & 54 Male & Private & Male & Forced flexion injury & 4 & None & $\mathrm{Nil}$ \\
\hline
\end{tabular}

WC, Workers Compensation; MVA, Motor Vehicle Accident

reconstruction/repair following perilunate dislocations where both the SL ligament and lunotriquetral (LT) ligament are damaged. We would not perform this procedure in established scapholunate advanced collapse (SLAC) or where fixed instability could not be reduced intraoperatively.

Since developing this technique in 2009 , we have performed this operation on over 40 patients with Geissler grade III or four IV injuries. We report on the first 11 consecutive patients who have received this technique and have been prospectively reviewed over a 12-24-month period (-Table 1). We excluded patients who had concomitant

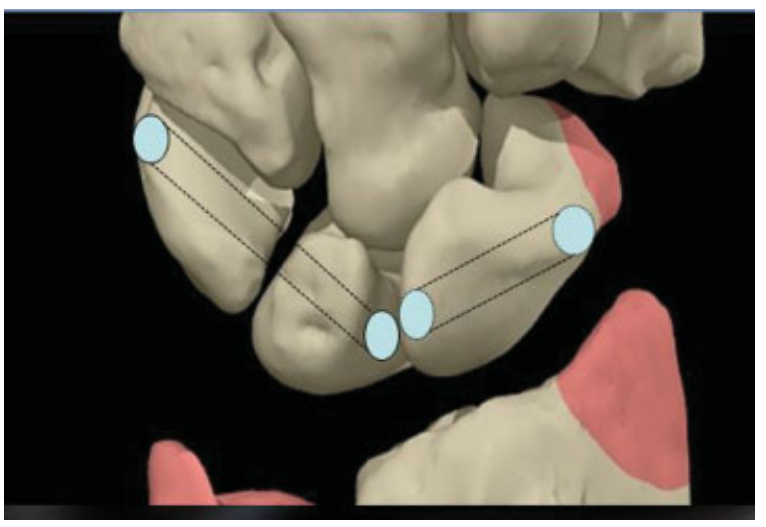

Fig. 1 Transosseous tunnel placement. trauma to the same upper extremity. The average follow up was 14 months (range 12-24 months). The average patient age was 36 years (range, $23-54$ years). $81.8 \%$ were male. The operative hand was dominant in $91 \%$ of patients. There was an average of 11.5 months from time of injury to surgery (range 4 to 30 months).

\section{Surgical Technique: Scapholunotriquetral Tenodesis (SLT)}

\section{Exposure}

A standard midline approach over the central dorsal aspect of the wrist is utilized.

A ligament-sparing Berger capsular flap ${ }^{1}$ is developed further to the ulnar side than is usual, to gain access to the ulnar border of the triquetrum immediately radial to the extensor carpi ulnaris (ECU) subsheath, which is not opened. In addition to elevation of the third and fourth compartments, division of the septum between the fourth and fifth compartments, and elevation of the extensor digiti minimi (EDM) tendon is required (-Fig. $\mathbf{2}$ ).

Exposure of the ulnar aspect of the triquetrum, drilling of the lunotriquetral (LT) tunnel, and graft tensioning can be facilitated by placing a small Hohmann retractor in the vicinity of the pisotriquetral joint. If there is a perilunate dissociation, the lunate and triquetrum should be reduced anatomically and pinned with a Kirschner wire (K-wire) 


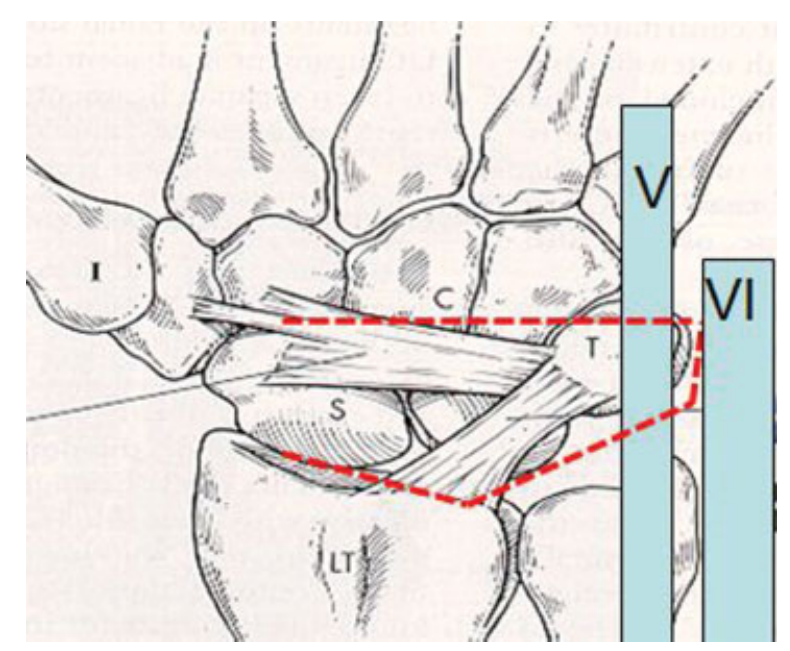

Fig. 2 Modified Berger flap.

before drilling the LT tunnel. Elevate the flap, stopping over the dorsal ridge of the scaphoid, thereby preserving the capsular attachments to the dorsum of the scaphoid, which carry the blood supply.

\section{Harvesting the Graft and Tunnel Preparation}

Volar exposure of the scaphoid is similar to the volar approach for scaphoid fixation and grafting described by Russe. ${ }^{2}$ Expose the tubercle and the distal half of the scaphoid. Minimize the division of the radioscaphocapitate ligament at this point. Harvest $\sim 40 \%$ of flexor carpi radialis (FCR) tendon using a technique allowing the tendon to be stripped from proximal to distal, leaving it attached distally. Tendon graft thickness should correspond to the transosseous tunnel diameter, which is usually $3 \mathrm{~mm}$. Once the portion of tendon is harvested, mobilize it until it is distal to the scaphotrapezoid (ST) joint.

\section{Scaphoid Tunnel}

Drill a hole using a 3-mm cannulated drill bit, in a process similar to a traditional Brunelli reconstruction, ${ }^{3,4}$ except that the entry point dorsally is on the articular facet of the scaphoid for the lunate (not at the dorsal insertion of the SL ligament). The tunnel should be just dorsal to the center point of lunate facet of the scaphoid. The exit point on the volar side of the scaphoid is a few millimeters proximal and radial to the normal point that would be used for a traditional dorsal band reconstruction ${ }^{5}$ ( $\mathbf{- F i g . ~ 2 )}$ ) The position of entry for the tunnel on the volar side needs to be accurate so that the tunnel can exit on the lunate facet of the scaphoid without violating the scaphocapitate joint just dorsal to the facet. The reason for this is that if the corresponding point on the facet of the lunate is volar to the midpoint, the tendon graft will travel obliquely, from dorsal on the scaphoid to volar on the lunate. This causes the graft to naturally reduce the rotary subluxation between the scaphoid and the lunate and correct the dorsal subluxation of the proximal pole of the scaphoid, as it is tensioned.

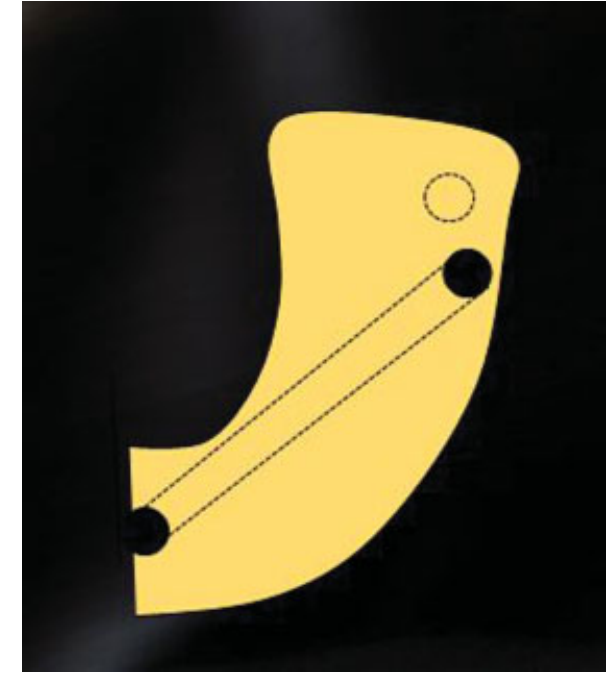

Fig. 3 Scaphoid tunnel placement.

\section{Lunotriquetral Tunnel}

The LT tunnel is drilled from the ulnar side of the triquetrum across the LT joint to exit on the lunate just volar to the midpoint of the articular facet. Care is taken not to make the entry point on the ulnar aspect of the triquetrum too dorsal to avoid the risk of fracturing the tunnel when the interference screw is inserted. Care must also be taken not to enter too proximally on the triquetrum, as the mediolateral dimension of the triquetrum decreases significantly in its more proximal portion, and the tunnel needs to be long enough to accommodate the 8-mm length of the interference screw without it protruding across the LT joint ( $\mathbf{- F i g . 3}$ ).

\section{Passing the Graft}

There are two methods of passing the graft: (1) place a lowprofile whip stitch in the end of the graft and pass the whip stitch through the tunnels with a wire loop; or (2) use a tendon passing device such as the Arthrex tendon passing device (Arthrex Inc., Naples, Florida).

\section{Reducing the Joint}

Pull on the tendon as it exits the triquetrum. The dorsal subluxation of the scaphoid will reduce and close the SL interval. Insert the interference screw (e.g., $3 \times 8$-mm polyetheretherketone [PEEK] screw, Arthrex Inc., Naples, Florida) into the triquetrum volar to the tendon. We have found that it is best to tension the graft with the wrist in ulnar deviation to ensure maximum tension. A similar biocomposite screw, such as tricalcium phosphate (TCP), would be preferable. We are reluctant to use a polylactic acid (PLA) screw because cyst formation in small carpal bones is probable.

Once the graft is secured, if there is any residual SL ligament tissue, it may be repaired using the surgeon's preferred technique. The graft is then passed across the dorsum of the midcarpal joint. The goal is to augment (or reconstruct) the dorsal intercarpal ligament. Place a small absorbable anchor with 2-0 suture in the waist of the scaphoid, being careful to avoid the tunnel containing the 
FCR graft. Suture the graft to the waist of the scaphoid. This is also the base of the Berger flap, so use either an artery forceps (hemostat) or tendon-braiding forceps to pass the graft through the base of the Berger flap.

\section{Closure}

To protect the reconstruction, a single $1.1-\mathrm{mm} \mathrm{K}$-wire is placed between the distal pole of the scaphoid and the capitate, or between the scaphoid and lunate, being careful not to perforate the graft. Surgeon preference may be to use two wires. The K-wire is left in situ for 6 to 8 weeks.

\section{Rehabilitation}

Postoperatively, the patient is allowed to mobilize the wrist to $30^{\circ}$ of flexion and $30^{\circ}$ of extension. If only one SL wire is used, oblique axis (dart-throwing) motion can be commenced early with the wire in situ. A custom-made thermoplastic wrist splint is worn at all other times until the K-wire is removed (i. e., 6-8 weeks). After the wire is removed, wrist mobilization exercises are upgraded to include orthogonal flexion/extension exercises and oblique dart-throwing-type exercises that focus on the midcarpal joint.

\section{Results}

We report on the results of 11 consecutive patients who have been tracked prospectively for a mean average of 14 months (range: 12-24). This cohort (representing the first 11 patients who received this procedure) demonstrated good early radiological and clinical outcomes for this new technique.

When the preoperative clinical results were compared with the most recent follow-up, the patients demonstrated pain relief with normal activities (Visual Analog Scale 1-100), improved Patient Rated Wrist Evaluation (PRWE), QuickDASH (Disabilities of the Arm, Shoulder, and Hand), range of movement (ROM, arc of extension to flexion using goniometric measurement), and grip strength (Jamar Dynamometer) scores. Radiological outcomes (SL angle and SL gap measured using X-ray) were improved.

Overall, QuickDASH scores improved from a preoperative mean of 50 (SD 15.22) to a mean of 21.2 (SD 15.33) postoperatively. The PRWE improved from 43.1(SD 7.25) to 18.92 (SD 7.25). The grips strength improved from a mean of $37.4 \mathrm{kgf}$ (SD 17.35) to $44 \mathrm{kgf}$ (SD 5.04) and pain with activity decreased from a mean of 17.8 (SD 6.54) to 13.56 (SD 13.99) on a visual analogue scale. The flexion-extension total arc of movement decreased minimally from $130.18^{\circ}$ (standard deviation 6.85) to $102.27^{\circ}$ (standard deviation 26.65).

The plain AP view $\mathrm{x}$-rays the SL interval decreased from a mean of $4.18 \mathrm{~mm}$ (standard deviation 0.603 ) to a mean of $1.627 \mathrm{~mm}$ (standard deviation 0.6604 ) at most recent follow up. Correspondingly, The SL angle decreased from $80.45^{\circ}$ (standard deviation 5.68) to $56.82^{\circ}$ (standard deviation 5.13).

In our cohort, we observed one patient who initially recovered well with good initial function and radiographic

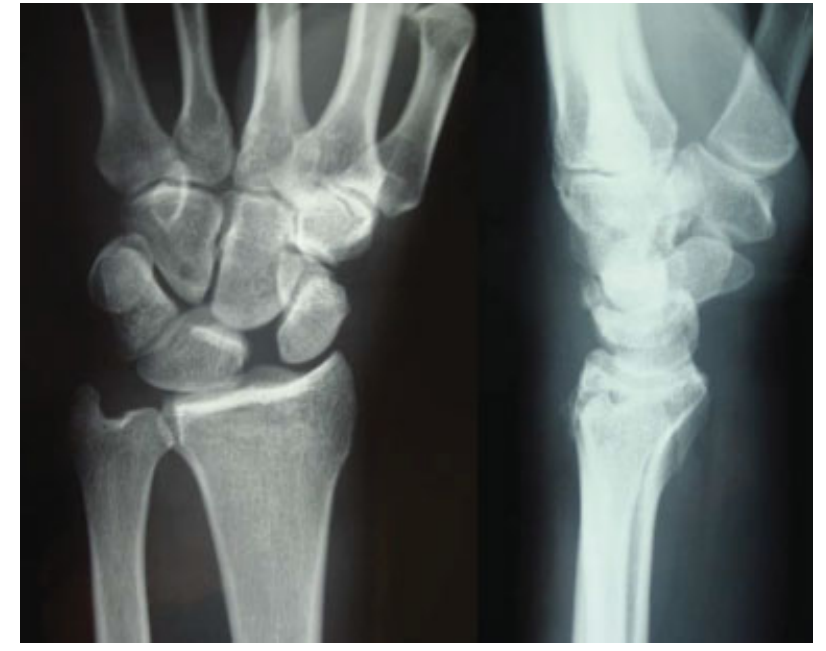

Fig. 4 Preoperative $X$-ray depicting scapholunate dissociation and undisplaced distal radius fracture of 40 -year-old male.

parameters and returned to professional football. At 10 months postsurgery, he suffered a high-energy hyperextension injury when driven into the ground hand first in a "spear" tackle. He sustained a fracture of the dorsal third of the lunate and was treated with a scaphocapitolunate fusion. Of note, this patient had larger (4-mm) tunnels and 4-mm interference screw. We would no longer use a 4-mm tunnel and remain unsure about the relative benefit and risk of a $3.5-\mathrm{mm}$ tunnel with a $4-\mathrm{mm}$ screw. Currently our preference is to use a 3-mm tunnel and a 3mm screw.

A case example of a reconstruction for a static deformity and distal radius fracture is illustrated in -Fig. 4 (preoperative X-ray) and $\boldsymbol{- F i g . ~} \mathbf{5}$ (6 months postoperative X-ray).

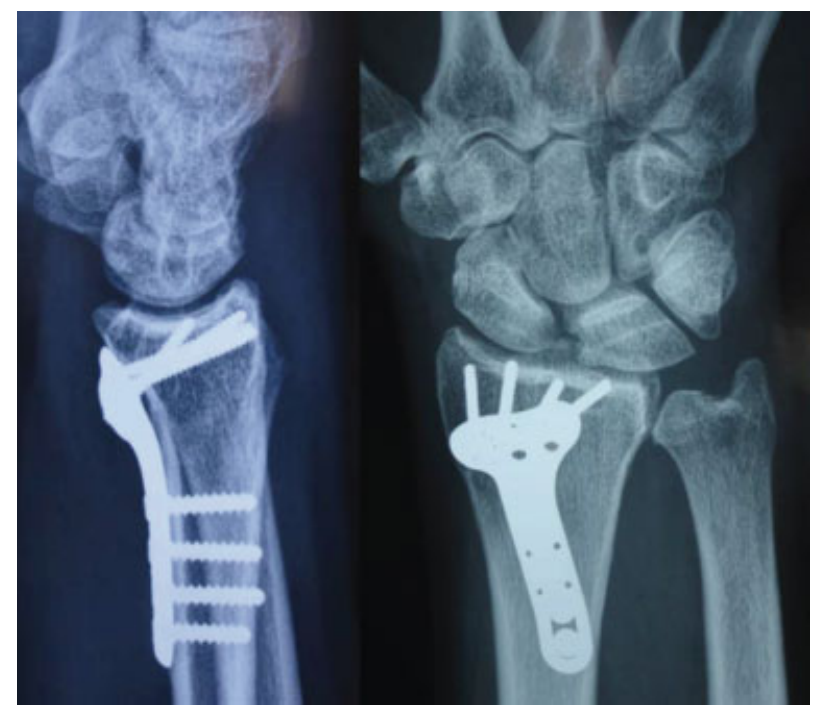

Fig. 5 Postoperative X-ray depicting ORIF distal radius fracture and scapholunate ligament reconstruction using the authors' technique. 


\section{Discussion}

Open reconstruction of the ruptured SL ligament can be accomplished by a variety of soft tissue and tenodesis techniques. Surgical reconstruction can be divided into procedures using tendons as grafts (such as FCR) and those using autologous free tissue grafts incorporating some variation of a "bone-soft tissue-bone" construct.

Weiss et $\mathrm{al}^{6}$ reported the bone-retinaculum-bone autograft technique of reconstruction in 1997 using bone between the second and third compartments and the overlying retinacular tissue.

Harvey and Hanel ${ }^{7,8}$ tested the scapholunate ligament in cadavers against bone-tissue-bone grafts, and the third metacarpal-carpal bone-tissue-bone technique was published in a clinical series in 2000.

The "modification" of the Brunelli ${ }^{3,4}$ technique popularized by Garcia-Elias and Stanley ${ }^{5}$ in 2006 as the three-ligament technique (3LT) eliminates the tether to the radius while reconstructing the dorsal radiotriquetral ligament as well as the ST and SL ligaments.

Our cohort is similar to those used in these studies, with an average age of 36 years compared with 34 for Weiss, ${ }^{6} 39$ for Harvey, ${ }^{8}$ and 31 for Garcia Elias. ${ }^{5}$ Our follow-up is shorter at average 14 months, compared with 3.6 years for Weiss, ${ }^{6} 2$ years for Harvey, and 46 months for GarciaElias. ${ }^{5}$

Our cohort had a higher percentage of static deformity preoperatively, with $72 \%$ compared with $21 \%$ for GarciaElias. ${ }^{5}$ Weiss reported the results of dynamic and static dissociation separately and reported significantly poorer outcomes for static deformity. Harvey ${ }^{8}$ simply commented that dynamic instability did better but did not specify numbers.

With regard to objective measures, our cohort had an average postoperative flexion-extension arc of $102^{\circ}$, which compared favorably to $103^{\circ}$ for Garcia-Elias. ${ }^{5}$ The dynamic group in Weiss's report ${ }^{6}$ showed $128^{\circ}$, but his static group was only $86^{\circ}$. Our average postoperative grip strength was $44 \mathrm{~kg}$, compared with $39 \mathrm{~kg}$ in Weiss's dynamic group ${ }^{6}$ and $22 \mathrm{~kg}$ in the static group. Garcia Elias ${ }^{5}$ reported grip as $65 \%$ of the contralateral side without an absolute figure, and Harvey $^{7,8}$ did not report grip.

Radiographic outcome is difficult to compare directly. In our series the SL interval (posteroanterior [PA] view) decreased from an average of $4.1 \mathrm{~mm}$ preoperatively to $1.6 \mathrm{~mm}$ at follow-up. None of the other authors directly reported preoperative scapholunate gaps. Weiss ${ }^{6}$ reported a gap of less than $3 \mathrm{~mm}$ in 13 out of 14 in the dynamic group, and an average gap of $5.8 \mathrm{~mm}$ in the static group. Harvey ${ }^{8}$ and Garcia-Elias ${ }^{5}$ did not report on this parameter. Our SL angle decreased from $80^{\circ}$ preoperatively to $56^{\circ}$ at final follow-up. Weiss reported follow-up angles of $50^{\circ}$ for the dynamic group and $64^{\circ}$ for the static group. Harvey ${ }^{8}$ did not report on SL angle, and Garcia-Elias ${ }^{5}$ reported on radiolunate angle instead, reporting only one patient out of 38 with a radiolunate angle greater than $25^{\circ}$.
Neither our cohort nor that of Garcia-Elias ${ }^{5}$ reported avascular necrosis of the scaphoid or lunate.

There was a paucity of subjective outcome measures to compare. The $\mathrm{PRWE}^{9}$ has been found to be a sensitive instrument for the evaluation of various wrist pathologies, and we have found it to be sensitive to change in this diagnostic group. Our cohort had a preoperative PRWE of 43, which decreased to 18 at final follow-up. No other authors reported on PRWE for this diagnosis.

We had a preoperative QuickDASH mean score of 50, which decreased to 21 . Only Harvey reported on the DASH, with a postoperative mean score of 28 . Neither Weiss ${ }^{6}$ nor Garcia-Elias ${ }^{5}$ reported outcome scores.

Notwithstanding our somewhat shorter follow-up, we believe that this comparison with these previously described techniques demonstrates that our technique achieves equivalent if not better subjective and objective outcomes. In addition, the percentage of higher-grade static deformity in our cohort presents a more challenging group of patients. However, this is not reflected in poorer outcome measures.

We began performing our technique in 2009 after extensive cadaveric dissection. We believe there are several benefits of our technique compared with these techniques. Furthermore, there are few techniques that address as many of the potential facets of SL pathology as this technique. The following are the main advantages of our technique:

1. In keeping with the initial intent of Brunelli's original description, ${ }^{3}$ our procedure provides a volar restraint to flexion of the distal pole of the scaphoid, and a reinforcement of the volar capsule of the STT joint.

2. There is no tethering of the dorsal carpus to the scaphoid. Therefore, there is no absolute restraint to radiocarpal flexion.

3. The tunnels are positioned at, or close to, the isometric point of rotation between the scaphoid and the lunate. As a consequence, the potential for sagittal-plane rotation between the scaphoid and the lunate is not restricted. In addition, as the graft is tensioned, there is even apposition of the scaphoid and the lunate facets. This avoids excessive tensioning dorsally, with consequent opening on the volar aspect, or vice versa, as may occur with volar or dorsal reconstruction or capsulodesis techniques.

4. As the graft is tensioned, there is automatic reduction of the major aspects of pathology, including the rotary dorsal subluxation of the proximal scaphoid and closure of the gap between the scaphoid and the lunate.

5. The transosseous passage of the graft avoids soft tissue bulk or formation of dorsal scar tissue, as occurs when a tendon graft is passed dorsally in the region of the dorsal band of the SL ligament and anchored to the lunate dorsally.

6. The triquetrum is ideally suited for anchoring of a tendon using an interference screw, and this anchoring in the triquetrum avoids excessive instrumentation of the lunate or scaphoid. 
7. Passage of the graft across the LT interval allows additional stabilization of the LT interval. Many of these injuries are part of a spectrum of perilunate injury, and as a consequence, there may be subtle recognized or unrecognized pathology affecting the LT ligament. As a consequence, this reconstruction is particularly suitable for reconstruction of complete perilunate injuries.

8. The secondary passage of the tendon graft across the dorsal aspect of the midcarpal joint back to the scaphoid reinforces and reconstructs the dorsal intercarpal ligament, which may also be secondarily involved in these patterns of injury. This secondary reconstruction also provides an additional reinforcement to the relationships of the proximal-row carpus in the coronal plane.

\section{Conclusion}

We can recommend use of this technique with caution in relation to the short nature of our follow-up and with regard to the complexity of this style of reconstruction (and careful adherence to the surgical technique). We would recommend practice of this technique in a cadaveric setting to ensure familiarity with the exposure and operative equipment requirements.

We are currently working on more minimally invasive exposure techniques and instruments to facilitate reproducible tunnel placement.

\section{Acknowledgments}

We thank Fraser Taylor (MBChB), Brisbane Hand and Upper Limb Research Institute and Princess Alexandra Hospital, Brisbane, Australia, and Susan Peters (BOccThy Hons), Brisbane Hand and Upper Limb Research Institute, for assistance with manuscript preparation and statistical analysis.

\section{Location}

The work was conducted through the Brisbane Hand and Upper Limb Research Institute, Brisbane, Queensland, Australia.

\section{Conflict of Interest \\ None}

\section{Declarations}

Interference screws for cadaveric preliminary investigation were donated by Arthrex Inc.

\section{References}

1 Berger RA, Bishop AT, Bettinger PC. New dorsal capsulotomy for the surgical exposure of the wrist. Ann Plast Surg 1995;35(1):54-59

2 Russe O. Fracture of the carpal navicular. Diagnosis, non-operative treatment, and operative treatment. J Bone Joint Surg Am 1960;42A:759-768

3 Brunelli GA, Brunelli GR. A new technique to correct carpal instability with scaphoid rotary subluxation: a preliminary report. J Hand Surg Am 1995;20(3 Pt 2):S82-S85

4 Brunelli GA, Brunelli GR. A new surgical technique for carpal instability with scapho-lunar dislocation.(Eleven cases)]. Annales de chirurgie de la main et du membre supérieur: organe officiel des sociétés de chirurgie de la main = Annals of hand and upper limb surgery 1995;14(4-5):207

5 Garcia-Elias M, Lluch AL, Stanley JK. Three-ligament tenodesis for the treatment of scapholunate dissociation: indications and surgical technique. J Hand Surg Am 2006;31(1):125-134

6 Weiss APC. Scapholunate ligament reconstruction using a boneretinaculum-bone autograft. J Hand Surg Am 1998;23(2):205-215

7 Harvey EJ, Hanel D, Knight JB, Tencer AF. Autograft replacements for the scapholunate ligament: a biomechanical comparison of hand-based autografts. J Hand Surg Am 1999;24(5):963-967

8 Harvey E, Hanel D. What is the ideal replacement for the scapholunate ligament in a chronic dissociation? Can J Plast Surg 2000;8:143-146

9 MacDermid JC, Turgeon T, Richards RS, Beadle M, Roth JH. Patient rating of wrist pain and disability: a reliable and valid measurement tool. J Orthop Trauma 1998;12(8):577-586 